EPJ Web of Conferences 43, 03009 (2013)

DOI: $10.1051 /$ epjconf/20134303009

(C) Owned by the authors, published by EDP Sciences, 2013

\title{
Mode lifetime and associated scaling relations
}

\author{
K. Belkacem¹,a , T. Appourchaux², F. Baudin², M.A. Dupret ${ }^{3}$, M.J. Goupil ${ }^{1}$, \\ J.P. Marques ${ }^{4}$, A. Noels ${ }^{3}$ and R. Samadi ${ }^{1}$ \\ ${ }^{1}$ LESIA, UMR8109, Observatoire de Paris, Université Pierre et Marie Curie, Université Denis \\ Diderot, 92195 Meudon Cedex, France \\ ${ }^{2}$ Institut d'Astrophysique Spatiale, CNRS, Université Paris XI, 91405 Orsay Cedex, France \\ ${ }^{3}$ Institut d'Astrophysique et de Géophysique, Université de Liège, Allée du 6 Août, 17, \\ 4000 Liège, Belgium \\ ${ }^{4}$ Georg-August-Universität Göttingen, Institut für Astrophysik, Friedrich-Hund-Platz 1, 37077 \\ Göttingen, Germany
}

\begin{abstract}
Thanks to the CoRoT and Kepler spacecrafts, scaling relations (linking seismic indices and global stellar parameters) are becoming the cornerstone of ensemble asteroseismology. Among them, the relation between the cut-off frequency and the frequency of the maximum in the power spectrum of solarlike pulsators as well as the relation between mode lifetime and the effective temperature remain poorly understood. However, a solid theoretical background is essential to assess the accuracy of those relations and subsequently of the derived stellar parameters. We will thus present recent advances on the understanding of the underlying mechanisms governing those relations and show that the physics of mode lifetime (thus of mode damping) plays a major role.
\end{abstract}

\section{INTRODUCTION}

The advent of space-borne asteroseismology, with the launch of CoRoT [1-3] and Kepler [4], gave birth to the ensemble asteroseismology. Its cornerstones are the relations between global seismic quantities (or seismic indices) and stellar parameters. It allows to infer model-independent stellar parameters as well as information on stellar structure and evolution. Scaling relations have initially been observationally derived, for solar-like oscillations, by several authors [5-7] using ground-based data. CoRoT and Kepler confirmed those results by providing accurate and homogeneous measurements for a large sample of stars including main sequence, subgiant, and red giant stars [8-14].

Among them, the relation between the frequency of the maximum height in the power spectrum $\left(v_{\max }\right)$ and the cut-off frequency $\left(v_{\mathrm{c}}\right)$ as well as the one between the mode line-width $(\Gamma)$ and the effective temperature $\left(T_{\text {eff }}\right)$ are particularly interesting since they both rely on the same cause: the physics of mode lifetime and thus non-adiabatic effects. The mode lifetime, which is proportional to the inverse of the mode line-width, results from the exchanges of energy between pulsations and the background. Consequently, those scaling relations give us additional constraints on those processes, still subject to many uncertainties.

In this paper, we will mainly discuss the physical origin of those relations and show how they are related to non-adiabatic processes. In Sect. 2, we address the $v_{\max }-v_{\mathrm{c}}$ relation and emphasize that it originates from the occurrence of the transition region in the super-adiabatic layers. In Sect. 3 , we

\footnotetext{
ae-mail: kevin.belkacem@obspm.fr
}

This is an Open Access article distributed under the terms of the Creative Commons Attribution License 2.0, which permits unrestricted use, distribution, and reproduction in any medium, provided the original work is properly cited. 


\section{EPJ Web of Conferences}

consider the relation between the mode line-width and the effective temperature. Finally, Sect. 4 is dedicated to conclusions.

\section{THE $v_{\text {MAX }}-v_{C}$ RELATION}

Solar-like oscillations are the result of a balance between mode driving and damping. Therefore, the frequency $v_{\max }$ is in principle determined by both physical mechanisms. To have a more precise view of what governs $v_{\max }$, one has to first determine what is responsible for the maximum height in the power spectrum. We thus consider the height $H$ of a given mode in the power spectrum, which is a natural observable. For stochastically excited modes, the height of the mode profile in the power spectrum is [15-17]

$$
H=\frac{P}{2 \eta^{2} \mathcal{M}},
$$

where $P$ is the excitation rate, $\eta$ the damping rate, and $\mathcal{M}$ the mode mass ${ }^{1}$. It turns out that the maximum of $H$ is determined by the plateau (or depression) of the line-widths. This is in agreement with theoretical computations [18-21] and recent observations of the solar-like stars by Kepler [22].

This depression originates from a destabilizing effect in the super-adiabatic layers and occurs when the modal period equals the thermal time-scale (or thermal adjustment time-scale) in the super-adiabatic layers. This was first mentioned by [23] and confirmed by [20] using two different non-adiabatic pulsation codes, which makes this conclusion quite secure. This can be translated into the following condition

$$
\mathcal{Q}=2 \pi v_{\max } \tau \sim 1
$$

with the inverse of the thermal time-scale defined as

$$
\frac{1}{\tau}=\frac{L}{4 \pi r^{2} \rho c_{v} T H_{p}}=\frac{1}{\tau_{\mathrm{conv}}}+\frac{1}{\tau_{\mathrm{rad}}}
$$

where $L$ is the luminosity, $r$ the radius, $\rho$ the local density, $c_{v}=(\partial U / \partial T)_{\rho}$ with $U$ the specific internal energy, $H_{p}$ the pressure scale height, $\tau_{\text {rad }}$ and $\tau_{\text {conv }}$ the radiative and convective thermal time-scales, respectively. Note that Eq. (3) is a local formulation of the thermal time-scale but a non-local one can also be defined as shown by [24]. It is also useful to note that contrary to the situation of classical pulsators, for which the envelope is dominated by the radiative transport of energy, Eq. (3) exhibits contributions of both radiative and convective fluxes.

Equation (2) refers to what is more commonly called the transition region in the context of opacitydriven pulsators $[25,26]$. It separates the quasi-adiabatic layers from the non-adiabatic ones. This transition region is an essential ingredient of the $\kappa$-mechanism in opacity-driven pulsators. In those stars, pulsations are destabilized by the perturbation of the opacity. But to be efficient, this destabilization must fulfill several conditions [26-28], e.g. the transition region must lie in an ionization region. Indeed, the destabilization occurs in the ionization region and if $\tau \ll \Pi_{o s c}$ (where $\Pi_{o s c}$ is the modal period) the thermal structure adapts so quickly that the flux is frozen. In the opposite situation, i.e. if $\tau \gg \Pi_{o s c}$, we are in the quasi-adiabatic regime. So the value of $\tau$ has to be close to the one of $\Pi_{\text {osc }}$ in the ionization region for the destabilization to be efficient and override damping terms. The situation is similar in solar-like pulsators, except that the destabilization by the perturbation of the opacity never dominates over damping terms and that the situation is more complex given the presence of convection which modifies the thermal time-scale (see Eq. 2).

\footnotetext{
1 The mode mass corresponds to the total amount of mass effectively moved by a given mode. It is defined as $\mathcal{M}=$ $\int_{0}^{M}|\xi|^{2} \mathrm{~d} m /|\xi(r=R)|^{2}$.
} 
Ageing Low Mass Stars: From Red Giants to White Dwarfs

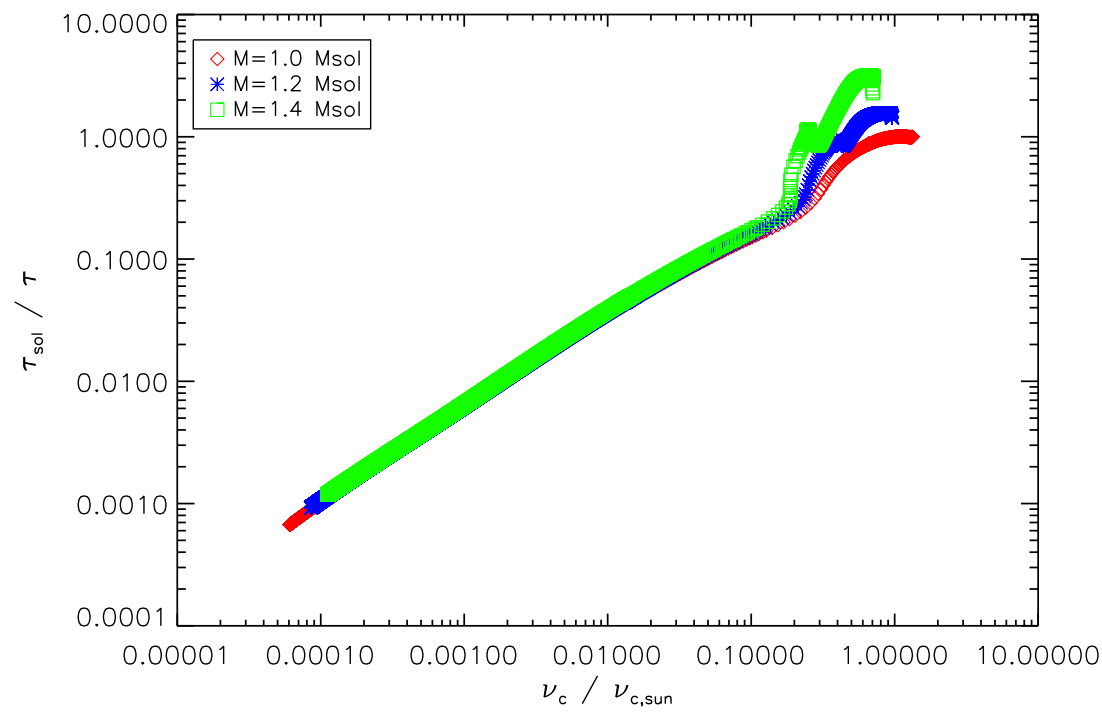

Figure 1. Thermal frequency $(1 / \tau)$ computed from Eq. (4) versus the cut-off frequency (computed as the ratio $c_{S} /(2 H p)$ ), normalized to the solar values, for models with different masses, i.e. $M=1.0 M_{\odot}, M=1.2 M_{\odot}$ and $M=1.4 M_{\odot}$, from the ZAMS to the ascending vertical branch. The input physics of the models can be found in [20].

We finally focus on the relation between the thermal time-scale $(\tau)$ and the cut-off frequency. In the mixing length theory framework the thermal time-scale expression can be recast as

$$
\frac{1}{\tau}=\frac{F_{\text {conv }}}{\rho c_{v} T H_{p}}\left[1+\frac{F_{\text {rad }}}{F_{\text {conv }}}\right] \propto\left(\frac{\mathcal{M}_{a}^{3}}{\alpha}\right)\left(\frac{c_{s}}{2 H_{p}}\right)\left[1+\frac{F_{\text {rad }}}{F_{\text {conv }}}\right],
$$

where $c_{s}$ is the sound speed, $\mathcal{M}_{a}=\mathrm{v}_{\text {conv }} / c_{s}$ the Mach number, and $\alpha$ the mixing length, $F_{\text {conv }}$ and $F_{\text {rad }}$ the convective and radiative fluxes respectively and $c_{s} /\left(2 H_{p}\right)$ the cut-off frequency.

This relation is verified, in Fig. 1, by using a grid of stellar models. The relation between the thermal time-scale and the cut-off frequency is very tight for red giants while a dispersion is observed for main sequence stars. Such a dispersion is explained by the dependence to the third power of the Mach. Indeed, this can be easily understood since the Mach number predominantly depends on the effective temperature that varies much more for main sequence and subgiant stars than for red giants. Equation (4) is useful to explicitly show the relation between $\tau$ and $v_{\mathrm{c}}$, but an investigation of this expression using a set of $3 \mathrm{D}$ hydrodynamic numerical simulations would be desirable in the future to get more quantitative estimates.

\section{THE $\Gamma-T_{\text {EFF }}$ RELATION}

For mode linewidths (or equivalently mode-damping rates), scaling relations have started to be investigated only very recently. This is the result of the need for long-time and almost-uninterrupted monitoring to resolve individual modes and to enable their precise measurements. [18] and later [29] investigated the dependence of mode-damping rates on global stellar parameters. From ground-based measurements, [29] found that observed mode linewidths follow a power-law of the form $\eta \propto T_{\text {eff }}^{4}$ and that no clear trend emerges when $\eta$ is scaled with the ratio $L / M$. Nevertheless, these measurements were based on short-term observations and derived from an inhomogeneous analysis and set of instruments, resulting in a large dispersion. This was settled by [9, 30] (Fig. 2) using a homogeneous sample of CoRoT data. They found that a unique power-law hardly describes the entire range of effective 


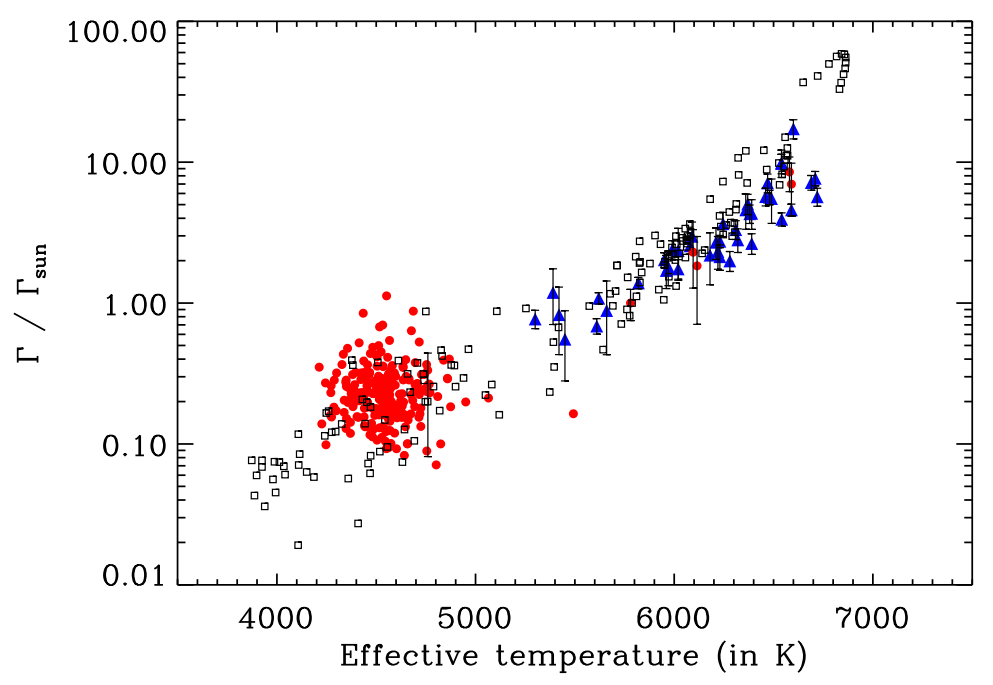

Figure 2. Mode linewidths (normalized by the solar value, $\Gamma_{\text {sun }}=0.95 \mu \mathrm{Hz}$ ) versus effective temperature. The squared symbols represent theoretical calculations computed as explained in [21]. The triangles correspond to the observations of main sequence stars derived by [22] from Kepler data (with their 3- $\sigma$ error bars). The dots correspond to the observations of red giants (with $T_{\text {eff }}<5200 \mathrm{~K}$ ) and main sequence stars (with $T_{\text {eff }}>5200 \mathrm{~K}$, with their $3-\sigma$ error bars) as derived by $[9,30]$ from CoRoT data.

temperature covered by main sequence and red giant stars. The latter result was later confirmed and extended to main sequence and subgiant stars [22] thanks to Kepler observations (Fig. 2). We also note that [31] proposed an exponential power law for mode linewidths. In absence of a strong theoretical argument to adopt either a power law or an exponential one, the statistical significance must dictate our choice and this is still to be performed. From a theoretical point of view, [29], based on the formalism developed by [18, 23] and [16], predicted a power-law of $\eta \propto T_{\text {eff }}^{4}$ which disagrees with CoRoT and Kepler observations [32]. In contrast, [21], based on the formalism of [33], were able to reproduce both CoRoT and Kepler observations.

To get more insight into the relation between $\eta$ and $T_{\text {eff }}$, let us first write down the integral expression of the damping rates [33]

$$
\eta=\frac{1}{2 \omega I} \int_{0}^{M} \mathcal{I} m\left[\frac{\delta \rho^{*}}{\rho_{0}} \frac{\delta P}{\rho_{0}}\right] \mathrm{d} m
$$

where $\omega$ is the mode frequency, $\delta \rho$ the Lagrangian perturbation of density, $\delta P$ the perturbation of the total pressure (including the turbulent pressure), $\rho_{0}$ the mean density, and where the star denotes the complex conjugate. The mode inertia is given by

$$
I=\int_{0}^{M}|\vec{\xi}|^{2} \mathrm{~d} m
$$

where $\vec{\xi}$ is the eigendisplacement vector. Therefore, a look at Eqs. (5) and (6) makes clear the need for disentangling the effects of mode inertia and work integral (i.e. the integral appearing in Eq. 5). The latter is related to non-adiabatic processes corresponding to a transfer of energy between pulsation and convection. Hence, it can be assumed at first glance that the work integral dimensionally scales as the 


\section{Ageing Low Mass Stars: From Red Giants to White Dwarfs}

ratio $L / M^{2}$. As verified by [21], it follows that the relation

$$
\eta I \propto\left(\frac{L}{M}\right)^{2.7}
$$

holds. In contrast, the mode inertia $(I)$ does not depend on mode energy leakage but on the static structure of the $\operatorname{star}^{3}$, and more precisely on the properties of its uppermost layers. Hence, one can expect mode inertia to scale as the surface gravity ${ }^{4}$. It has been shown in [21] that

$$
I \propto g^{-2.4} \text {. }
$$

Using Eq. (7) and Eq. (8), it turns out that

$$
\eta \propto T_{\mathrm{eff}}^{10.8} g^{-0.3}
$$

This simple analysis allows to explain qualitatively the strong dependence of mode damping rates on effective temperature.

To confront Eq. (9) with recent Kepler observations of main sequence stars, let us consider the relation

$$
\Gamma \propto T_{\mathrm{eff}}^{8.75-4 \beta} g^{\beta}
$$

where $\beta$ is a parameter to be determined ${ }^{5}$. This is performed by minimizing a $\chi^{2}$, and it gives

$$
\begin{aligned}
& \beta_{1}=-0.45 \pm 0.29 \\
& \beta_{2}=-0.50 \pm 0.26 \\
& \beta_{3}=-0.35 \pm 0.25
\end{aligned}
$$

where $\beta_{1}$ is obtained using the effective temperatures from [34], $\beta_{2}$ from [35, 36], and $\beta_{3}$ from Kepler Input Catalog (KIC). Those results show that Eq. (9) is compatible with current Kepler observations. However, it is clear that the limiting factor remains the high uncertainties on effective temperatures.

\section{CONCLUSIONS}

We have shown that two scaling relations $\left(v_{\max }-v_{\mathrm{c}}\right.$, and $\left.\Gamma-T_{\mathrm{eff}}\right)$ rely on the physics of mode damping rates. Up to very recently, those relations were not understood. The basic physical picture is now grasped $[20,21]$. The remaining uncertainties are mainly related to the complexity of non-adiabatic processes involving time-dependent treatment of convection but do not come from the failure of the physical picture.

Further investigation of those scaling relations is nevertheless highly desirable. We need to go deeper into the $v_{\max }-v_{\mathrm{c}}$ relation, so that we can improve it. This is particularly important to derive seismic stellar parameters such as the mass and the radius. Concerning the relation between $\Gamma$ and $T_{\text {eff }}$, an improvement of the relation is also important since one can potentially use it to derive the effective temperature, and with the use of the seismic indices $\left(v_{\max }, \Delta v\right.$, and $\Gamma$ ) one would be able to derive the mass, the radius, and effective temperatures of the star from seismology only and without the help of stellar modeling.

Such a long-term objective must motivate us to further improve our understanding of non-adiabatic processes in solar-like stars, and more precisely the coupling between pulsation and convection.

\footnotetext{
2 This comes from the perturbed energy equation that shows that entropy perturbation dimensionally scales as the ratio $L / M$.

${ }^{3}$ Except through non-adiabatic effects on mode eigendisplacement.

${ }^{4}$ Note that mode inertia also scales as the dynamical timescale $\left.\sqrt{(} G M / R^{3}\right)$ with almost the same dispersion as for the surface gravity.

${ }^{5}$ The parameter $\beta$ permits us to measure the departure from a scaling of $T_{\mathrm{eff}}$ only. In other words, the parameter $\beta$ measures the departure from a perfect compensation of gravity between Eqs. (7) and (8).
} 
EPJ Web of Conferences

\section{References}

[1] A. Baglin, M. Auvergne, P. Barge, M. Deleuil, C. Catala, E. Michel, W. Weiss, COROT Team, Scientific Objectives for a Minisat: CoRoT, in ESA Special Publication, edited by M. Fridlund, A. Baglin, J. Lochard, L. Conroy (2006), Vol. 1306 of ESA Special Publication, p. 33

[2] A. Baglin, M. Auvergne, L. Boisnard, T. Lam-Trong, P. Barge, C. Catala, M. Deleuil, E. Michel, W.Weiss, CoRoT: a high precision photometer for stellar ecolution and exoplanet finding, in 36th COSPAR Scientific Assembly (2006), Vol. 36 of COSPAR Meeting, p. 3749

[3] E. Michel, A. Baglin, M. Auvergne, C. Catala, R. Samadi, F. Baudin, T. Appourchaux, C. Barban, W.W. Weiss, G. Berthomieu et al., Science 322, 558 (2008), 0812.1267

[4] W.J. Borucki, D. Koch, G. Basri, N. Batalha, T. Brown, D. Caldwell, J. Caldwell, J. ChristensenDalsgaard, W.D. Cochran, E. DeVore et al., Science 327, 977 (2010)

[5] R.K. Ulrich, ApJ 306, L37 (1986)

[6] T.M. Brown, R.L. Gilliland, R.W. Noyes, L.W. Ramsey, ApJ 368, 599 (1991)

[7] H. Kjeldsen, T.R. Bedding, A\&A 293, 87 (1995), arXiv: astro-ph/9403015

[8] B. Mosser, K. Belkacem, M.J. Goupil, A. Miglio, T. Morel, C. Barban, F. Baudin, S. Hekker, R. Samadi, J. De Ridder et al., A\&A 517, A22 (2010), 1004.0449

[9] F. Baudin, C. Barban, K. Belkacem, S. Hekker, T. Morel, R. Samadi, O. Benomar, M.J. Goupil, F. Carrier, J. Ballot et al., A\&A 529, A84 (2011), 1102.1896

[10] B. Mosser, C. Barban, J. Montalbán, P.G. Beck, A. Miglio, K. Belkacem, M.J. Goupil, S. Hekker, J. De Ridder, M.A. Dupret et al., A\&A 532, A86 (2011), 1105.6113

[11] B. Mosser, K. Belkacem, M.J. Goupil, E.Michel, Y. lsworth, C.Barban, T. allinger, S. Hekker, J.De Ridder, R. amadi et al., A\&A 525, L9 (2011), 1011.1928

[12] B. Mosser, M.J. Goupil, K. Belkacem, E. Michel, D. Stello, J.P. Marques, Y. Elsworth, C. Barban, P.G. Beck, T.R. Bedding et al., A\&A 540, A143 (2012), 1203.0689

[13] B. Mosser, Y. Elsworth, S. Hekker, D. Huber, T. Kallinger, S. Mathur, K. Belkacem, M.J. Goupil, R. Samadi, C. Barban et al., A\&A 537, A30 (2012), 1110.0980

[14] R. Samadi, K. Belkacem, M.A. Dupret, H.G. Ludwig, F. Baudin, E. Caffau, M.J. Goupil, C. Barban, A\&A 543, A120 (2012), 1205.4846

[15] F. Baudin, R. Samadi, M.J. Goupil, T. Appourchaux, C. Barban, P. Boumier, W.J. Chaplin, P. Gouttebroze, A\&A 433, 349 (2005)

[16] W.J. Chaplin, G. Houdek, Y. Elsworth, D.O. Gough, G.R. Isaak, R. New, MNRAS 360, 859 (2005)

[17] K. Belkacem, R. Samadi, M.J. Goupil, F. Kupka, F. Baudin, A\&A 460, 183 (2006), arXiv:astro-ph/0607570

[18] G. Houdek, N.J. Balmforth, J. Christensen-Dalsgaard, D.O. Gough, A\&A 351, 582 (1999), arXiv: astro-ph/9909107

[19] W.J. Chaplin, G. Houdek, T. Appourchaux, Y. Elsworth, R. New, T. Toutain, A\&A 485, 813 (2008), 0804.4371

[20] K. Belkacem, M.J. Goupil, M.A. Dupret, R. Samadi, F. Baudin, A. Noels, B. Mosser, A\&A 530, A142 (2011), 1104.0630

[21] K. Belkacem, M.A. Dupret, F. Baudin, T. Appourchaux, J.P. Marques, R. Samadi, A\&A 540, L7 (2012), 1203.1737

[22] T. Appourchaux, O. Benomar, M. Gruberbauer, W.J. Chaplin, R.A. García, R. Handberg, G.A. Verner, H.M. Antia, T.L. Campante, G.R. Davies et al., A\&A 537, A134 (2012), 1112. 3295

[23] N.J. Balmforth, MNRAS 255, 603 (1992)

[24] W.D. Pesnell, Ph.D. thesis, Florida Univ., Gainesville. (1983)

[25] J.P. Cox, Reports on Progress in Physics 37, 563 (1974)

[26] J.P. Cox, Theory of stellar pulsation (1980)

[27] J.P. Cox, R.T. Giuli, Principles of stellar structure (1968) 
[28] A.A. Pamyatnykh, Acta Astron. 49, 119 (1999)

[29] W.J. Chaplin, G. Houdek, C. Karoff, Y. Elsworth, R. New, A\&A 500, L21 (2009), 0905.1722

[30] F. Baudin, C. Barban, K. Belkacem, S. Hekker, T. Morel, R. Samadi, O. Benomar, M.J. Goupil, F. Carrier, J. Ballot et al., A\&A 535, C1 (2011)

[31] E. Corsaro, D. Stello, D. Huber, T.R. Bedding, A. Bonanno, K. Brogaard, T. Kallinger, O. Benomar, T.R. White, B. Mosser et al., ApJ 757, 190 (2012), 1205.4023

[32] G. Houdek, ArXiv e-prints (2012), 1201.0194

[33] A. Grigahcène, M.A. Dupret, M. Gabriel, R. Garrido, R. Scuflaire, A\&A 434, 1055 (2005)

[34] M.H. Pinsonneault, D. An, J. Molenda-Żakowicz, W.J. Chaplin, T.S. Metcalfe, H. Bruntt, ApJS 199, 30 (2012), 1110.4456

[35] L. Casagrande, L. Portinari, C. Flynn, MNRAS 373, 13 (2006), arXiv: astro-ph/0608504

[36] L. Casagrande, I. Ramírez, J. Meléndez, M. Bessell, M. Asplund, A\&A 512, A54 (2010), 1001.3142 